Revista de Psicología de la PUCP. Vol. XIX, 2, 2001

\title{
Predictores familiares y conductuales de la problemática escolar en alumnos de secundaria y preparatoria $^{1}$
}

\author{
Martha Frías Armenta ${ }^{2}$, Víctor Corral Verdugo ${ }^{3}$, Amelia López Escobar $^{4}$, \\ Sylvia Díaz Méndez $z^{5}$ y Erica Peña Bustamante \\ Universidad de Sonora
}

\begin{abstract}
Se presenta un modelo de influencias familiares en el desarrollo de conducta antisocial, delicti$\mathrm{va}$, rendimiento y problemas escolares en adolescentes. Doscientos cuatro estudiantes de educación secundaria y preparatoria fueron evaluados y sus respuestas se analizaron en un modelo estructural. Los resultados mostraron que el maltrato de los padres, el vivir en una familia no cooperativa y la ingesta de alcohol y drogas de la madre influía en el desarrollo de conducta antisocial, la cual promovía el comportamiento delictivo y afectaba negativamente el promedio escolar. La conducta delictiva y la ingesta de alcohol de la madre influían en los problemas escolares, los cuales podían ser parcialmente revertidos por las habilidades sociales de los jóvenes. Se observó la necesidad de orientación escolar a las familias para prevención.

Palabras clave: familia, conducta antisocial, problemas escolares, adolescentes.
\end{abstract}

Family and behavioral predictors of school problems in junior and high school students A model of family influences on the development of antisocial behavior and scholar problems in adolescents is presented. Two-hundred four students of junior and high school were assessed. Data were analyzed through a structural equation model. Results showed that child abuse, a nocooperative family and mothers' alcohol consumption had a direct effect on antisocial behavior, which in turn promoted delinquent behavior and negatively affected school grades of students. Delinquency and mothers' alcohol consumption had an influence on students' school problems, which could be partially overturned by their social abilities. Results suggest the necessity of counselling for families in order to prevent school problems and bad grades in adolescents.

Keywords: family, anti-social behavior, school problems, adolescents.

1 Esta investigación se desarrolló gracias a los auspicios del Consejo Nacional de Ciencia y Tecnología (CONACyT) de México (Proyecto 35166-H).

2 Profesora titular. Doctora en Psicología por la Universidad de Arizona. Ha realizado investigaciones sobre la violencia familiar, delincuencia juvenil y familia y educación. Correo electrónico: marthafrias@sociales.uson.mx.

3 Profesor titular. Doctor en Psicología por la Universidad de Arizona. Su línea de interés está relacionada con las conductas protectoras del ambiente, la violencia intrafamiliar y los modelos conductuales de capacidades conductuales.

4-5-6 Estudiantes del Departamento de Psicología de la Universidad de Sonora. 

La problemática escolar ha sido tradicionalmente estudiada desde la individualidad del estudiante. En la visión popular, los alumnos no aprenden o se comportan mal en la escuela debido a problemas intrínsecos de personalidad. De acuerdo con la literatura relevante, es posible que estas variables afecten pero, además, existen otras que pudieran influir igualmente en este fenómeno y, dentro de ellas, la familia constituye un factor de orden primordial (Dabas, 1998). La familia es el escenario en el que lo(a)s niño(a)s aprenden la forma de interactuar con el mundo circundante, incluyendo al mundo del barrio, la comunidad y la escuela. Desde esta perspectiva resultan de fundamental importancia las interacciones familiares en el desarrollo del(a) niño(a) (Frías, Corral, Moreno y Rodríguez, 2000).

Varios investigadores mencionan que la interacción entre los factores biológicos y los factores de crianza en la génesis de los problemas personales es difícil de entender (Garbarino y Eckenrode, 1999). Además, existe una discusión acerca de qué es lo que determina la conducta de los individuos. Por un lado están los factores biológicos y por el otro, la influencia del ambiente. Algunos autores indican que los factores biológicos son más importantes en el desarrollo de la conducta de los individuos. Sin embargo, aun estos autores reconocen la interdependencia entre la biología y el ambiente; esto es, que la conducta de los individuos está determinada tanto por la herencia biológica como la familia y la sociedad en donde se desenvuelven. La conducta de los seres humanos no depende únicamente de las características biológicas de las personas, sino que también influyen en esta los contextos en los que se desenvuelven los individuos (Bronfenbrenner, 1979). El medio principal en donde se desarrolla el (la) niño(a) es la familia. Al principio, para la mayoría de los menores sus interacciones ocurren en un medio muy reducido, el hogar, y estas se llevan a cabo con una sola persona a la vez (Garbarino y Eckenrode, 1999). A medida que el (la) niño(a) crece, aumenta la complejidad de sus contactos, 
y las interacciones son con más cosas, lugares y personas. Una de las fuentes de problemas conductuales en los individuos consiste en la escasa gama de actividades en esta etapa del desarrollo.

La violencia en el hogar, tanto el maltrato dirigido hacia los niños como el maltrato hacia las madres de estos, resulta en un empobrecimiento del ambiente familiar. Dicho empobrecimiento causa que los niños presenten problemas en su desarrollo, manifestándose en la escuela cuando estos ingresan a ella. Por otro lado, la violencia puede tener consecuencias devastadoras en los menores, las que pueden manifestarse como conducta antisocial o autodestructiva en los niños (Straus, 1991).

Las secuelas podrían manifestarse también en el bajo aprovechamiento escolar o en conductas problemáticas de los menores. Por ejemplo, en un estudio llevado a cabo por Anderson y Walsh (1998) se encontró que jóvenes con baja eficiencia verbal provenían de hogares con bajas oportunidades de mejorar su capacidad lingüística y mostraron más conducta antisocial y delictiva cuando fueron adolescentes y adultos. Es probable que este efecto se deba a que la familia no sea capaz de dotar a los menores de las capacidades necesarias de interacción prosocial (conseguir un buen empleo, interactuar de manera positiva con otras personas) y esto los lleve a conseguir sus satisfactores de manera antisocial o delictiva (Hunter, comunicación personal, 5 de julio de 2000).

La familia ocupa un papel central en el desarrollo de la personalidad del menor. La delincuencia juvenil, según algunos autores, es una de las consecuencias negativas que surgen de las condiciones familiares inadecuadas (Gove y Crutchfield, 1982; Loeber, 1986). Uno de los reportes más frecuentemente encontrados en los estudios criminólogos es que las circunstancias familiares adversas contribuyen a causar y mantener la conducta delictiva (Wells y Rankin, 1986). Loeber y Dishion (1982) identificaron al funcionamiento familiar y las prácticas paternas de crianza como unas de las mejores predictoras de la delincuencia juvenil. En la estructura de la familia del joven delincuente 
Predictores familiares y conductuales de la problemática escolar en alumnos...

se presenta una clara imagen de una paternidad deficiente y de modelos inadecuados. Así, se describen cuatro factores de la paternidad asociados al desarrollo de la delincuencia en los jóvenes:

- Los delincuentes juveniles provienen de hogares desintegrados; sin embargo, no siempre ese tipo de familias provoca la delincuencia.

- La calidad de las relaciones entre los padres y los hijos es fundamental: si la relación es cálida y afectuosa, el índice de delincuencia juvenil baja. Los adolescentes que provienen de familias con mucha hostilidad y poco afecto son más propensos a presentar antecedentes penales. Los niños criados en hogares hostiles tienden a ser agresivos, desinteresados en los derechos y sentimientos de los demás y no son cooperativos. De la misma manera, la calidad de la relación hijos-padres también se asocia a la conducta delictiva. Así, mientras más apoyo brinden los padres a los adolescentes, menor es la probabilidad de cometer actos delictivos.

- El tercer factor se relaciona con el uso de la disciplina. Los padres de los adolescentes problemáticos emplean la fuerza, aplican o amenazan con el castigo físico, la disciplina es drástica y se caracteriza por la pérdida del control emocional de los padres, la exhibición irracional de la fuerza y las palizas repentinas y drásticas. El castigo es inconsistente, con una manifestación errática que combina restricción y tolerancia.

- La conducta criminal y el alcoholismo del padre, en particular, han sido algunos de los factores más potentes en el riesgo de la conducta criminal; el uso de una disciplina laxa, caprichosa e inconstante por parte de uno o de ambos padres ha demostrado estar relacionada con la conducta delictiva (Kazdin 1988; Sarafino y Amstrong 1991).

Por otro lado, Angenent y De Man (1996), después de analizar una serie de estudios relacionados con el funcionamiento escolar y la delincuencia juvenil, señalan que existen tres explicaciones para esta relación. La primera se refiere a la delincuencia juvenil como 
una reacción ante el pobre funcionamiento escolar, posteriormente se plantea una relación inversa donde es la delincuencia la que influye en el bajo rendimiento escolar; el menor delincuente dispone de menos tiempo y energía para dedicar a tareas escolares. Por último, los autores señalan que en la mayoría de los casos la relación entre el bajo funcionamiento escolar y la delincuencia se debe a causas mucho más profundas que engloban a ambos problemas al mismo tiempo (causas de bagaje individual y desarrollo personal, como el síndrome de desviación comportamental).

Los estudios anteriormente mencionados señalan que la violencia dentro del hogar genera conducta antisocial en los menores, y la conducta antisocial provoca que los niños tengan un bajo aprovechamiento escolar y problemas de conducta en la escuela. Un factor importante en la salud mental de los menores es que se sientan queridos y respetados por sus padres. Los que sienten que sus padres los aprecian tienen más probabilidad de defender sus sistemas de valores prosociales y de cuidar su propia imagen social (Garbarino y Eckenrode, 1999).

En el mismo sentido, el desarrollo del sentimiento de autoeficacia depende primordialmente de la aprobación de las personas que son significativas para los menores, particularmente de los padres (Harter, 1998). Los sentimientos negativos y la carencia de habilidades sociales en los niños maltratados pudieran derivarse de la falta de apoyo y aprobación de los padres. La ausencia de estas habilidades en los niños o la percepción de que las carecen (por parte de los niños) ha sido relacionada con el bajo aprovechamiento escolar (Kinard, 2001). En otros estudios se ha encontrado que los niños maltratados obtienen un aprovechamiento escolar más bajo que los niños que no han sido maltratados (Frías, Corral, Moreno y Rodríguez, 2000; Kinard, 1999). Igualmente, se ha encontrado que algunas víctimas de violencia severa experimentan un alto índice de reprobaciones y problemas disciplinarios (Eckenrode y Doris, 1991; Gelles y Straus, 1990). 
Considerando estos antecedentes, el propósito del presente trabajo fue el de estudiar factores familiares que pudieran afectar el desempeño escolar de jóvenes estudiantes de secundaria y preparatoria en una comunidad mexicana. Con este fin, se recogió información acerca de esas variables, la cual se analizó dentro de un modelo estructural. En ese modelo se especificó que las variables familiares tenían un efecto en el desarrollo de conducta positiva (asertividad, habilidades sociales) y comportamiento negativo (conducta antisocial y delictiva), los cuales a su vez afectaban al desempeño y a los problemas escolares.

\section{Metodología}

\section{Paticipantes}

Se entrevistaron 204 jóvenes que cursaban la educación secundaria y preparatoria, con una media de edad de 15 años. El promedio escolar de los estudiantes entrevistados fue de 64 y la desviación estándar fue de 33, la misma proporción de los participantes fueron del sexo masculino y femenino (50\% y $50 \%$ por c/u). El Cuadro 1 nos muestra las principales características demográficas de la muestra.

\section{Cuadro 1}

Medias de variables demográficas

\begin{tabular}{|lccrcc|}
\hline Variables & $N$ & Media & $D E$ & Mínimo & Máximo \\
\hline Edad & 204 & 15.00 & 1.70 & 12 & 19 \\
Promedio escolar & 204 & 64.80 & 33.49 & 0 & 99 \\
Edad madre & 173 & 38.00 & 3.35 & 30 & 41 \\
Edad padre & 204 & 41.00 & 4.61 & 35 & 47 \\
Ingreso familiar (US\$) & 200 & 306.40 & 76.70 & 0 & 5000 \\
\hline
\end{tabular}

Las escuelas se seleccionaron tomando en cuenta los parámetros del Instituto Nacional de Geografía e Informática (INEGI, 1992). El INEGI organizó el sistema estadístico nacional en áreas geográficas a 
tres niveles: área Geoestadística Estatal (AGEE), área Geoestadística Municipal (AGEM), y área Geoestadística Básica (AGEB). Los AGEB constituyen la unidad fundamental del marco geoestadístico nacional y constituyen una selección de los principales conceptos de la temática censal en la cual es plausible calcular indicadores específicos, y en general, realizar operaciones para obtener estadísticas derivadas. INEGI define AGEB como lo siguiente:

[...] el espacio geográfico delimitado mediante rasgos naturales o culturales, permanentes y reconocibles en el terreno, con extensión convencional al interior de cada Municipio del País o Delegación del Distrito Federal que facilita la capacitación y referenciación geográfica de la información. (INEGI, 1992)

Para el estudio, se seleccionó un AGEB que fuera representativo de los tres estratos socioeconómicos (alto, medio, y bajo) de la ciudad y después se seleccionaron las escuelas que representaran ese AGEB. Una vez determinadas las escuelas, se entrevistó un grupo de cada grado; en la escuela secundaria, se entrevistó un grupo de primero, uno de segundo y uno de tercero. En la escuela preparatoria se entrevistó un grupo de primer semestre, uno de tercero y uno de quinto.

\section{Instrumentos}

Se aplicó una batería de pruebas consistente en 10 escalas, 6 de las cuales fueron elaboradas en los Estados Unidos, traducidas al español y piloteadas con anterioridad al presente estudio; las otras 4 escalas fueron elaboradas expresamente para esta investigación.

Maltrato y castigo de los padres. Esta escala contiene diez ítems que fueron tomados de la Escala de Tácticas de Conflicto de Straus (1979; Straus, 1990; Straus 1998) y fue administrada a todos los niños de la muestra; estos reactivos miden la frecuencia de la violencia 
Predictores familiares y conductuales de la problemática escolar en alumnos...

física y emocional del padre y de la madre hacia sus hijos, en una escala de cero a cinco $(0=$ nunca, $1=$ una vez, $2=$ dos veces, $3=$ de tres a cinco veces, $4=$ seis a diez veces, $5=$ once a veinte veces, $6=$ más de veinte veces). Straus reportó un alfa de Cronbach de .72 de los trece ítems utilizados.

Violencia entre los padres. Se utilizaron 12 ítems de la escala de violencia intramarital de Straus (1980). Esta se contesta empleando una escala que va de 0 a $6(0=$ nunca... $6=$ más de veinte veces) al igual que en la escala de los niños, se obtuvo un alfa de .91 de los reactivos utilizados.

Asertividad. Esta medida consta de once reactivos; mide el grado de acuerdo o desacuerdo del niño con ciertas conductas que indican asertividad ( $0=$ está /estaría totalmente en desacuerdo, 1=está/estaría parcialmente en desacuerdo, $2=$ no está de acuerdo ni en desacuerdo, $3=$ está parcialmente de acuerdo y $4=e s t a ́$ totalmente de acuerdo. Esta escala fue desarrollada por Grasmick, Tittle, Bursik y Bruce (1993).

Conducta antisocial y delictiva. Está constituida por 17 preguntas que miden el número de veces que el niño ha cometido un acto o conducta antisocial o delictiva especificada en el instrumento, durante los 12 meses anteriores a la entrevista $(0=$ nunca, $1=$ una o dos veces, $2=$ de tres a cinco veces, $3=$ de seis a diez veces, $4=$ de once a veinte veces y $5=$ más de veinte veces). Las acciones reflejan el número de veces que los menores reportaron haber golpeado a alguien, robado cosas, o hecho daños en propiedad ajena en el último año. En un estudio anterior se reportó un alfa de .75 (Frías, Ramírez, Soto, Castell y Corral, 2000).

Habilidades sociales. Para medir las habilidades sociales del menor se utilizó la escala elaborada por Goldstein et al. (Goldstein, Sprafkin, Gershaw y Klein, 1989) la cual fue modificada para este estudio. Esta medida contenía 19 preguntas tipo Likert de cinco puntos 
$(0=$ nunca, $1=$ casi nunca, $2=$ regularmente, $3=$ casi siempre y $4=$ siempre), de la que se obtuvo un alfa de 83 .

Uso de alcohol de la madre. El uso de alcohol y drogas fue medido con preguntas obtenidas del Inventario de Diagnóstico de Alcohol para Padres (Reich y Herjanic, 1989). Se utilizaron tres ítems del inventario, los cuales median la frecuencia y la cantidad de alcohol que ingería la madre por semana. La frecuencia se midió en una escala de 6 puntos $(0=$ nunca... $5=$ todos los días de la semana) y la cantidad en una escala de 5 puntos $(0=$ nada... $4=$ más de seis bebidas en cada ocasión).

Problemas escolares. Los problemas escolares fueron medidos en una escala que fue elaborada por Frías, Corral, Moreno y Rodríguez (2000). En esta escala los niños reportaron el número de veces que manifestaron una conducta inapropiada (indisciplina) en la escuela en una escala de 6 puntos $(0=$ nunca, $1=$ una o dos veces... y $5=$ más de 20 veces).

Clima familiar. Estas preguntas fueron elaboradas expresamente para el presente estudio, en donde los niños reportaban la cohesión y la cooperación dentro de la familia. Se obtuvo una consistencia interna de .77 .

\section{Procedimiento}

Los alumnos contestaron el cuestionario en el aula en donde recibían clases. Dos psicólogas clínicas estuvieron aclarando sus dudas y dando las instrucciones dentro del aula. A los alumnos se les informó que su participación era totalmente voluntaria y que podían dejar de contestar el cuestionario en cualquier momento. De la misma manera se les aseguró total confidencialidad de la información que se obtuviera. 
Los datos fueron analizados en el programa estadístico SAS, de donde se obtuvieron estadísticas univariadas, como son las frecuencias de las variables categóricas, las medias y las desviaciones estándar de las variables continuas y las alfas de Cronbach de las escalas utilizadas. Los promedios de las escalas constituyeron índices que representaron a las variables a relacionar. Con estos índices se elaboró la matriz de correlaciones para probar el modelo propuesto en otro programa estadístico.

Posteriormente, se probó el modelo teórico a través de un análisis de trayectorias, utilizando el paquete EQS (Bentler, 1993). El análisis de trayectorias es la técnica estadística que nos permite modelar y estimar un sistema completo de relaciones causales entre diversos componentes, los cuales podrán ser variables dependientes e independientes a la vez. La ventaja de este sistema es que se pueden medir al mismo tiempo los efectos directos e indirectos que tienen una variable sobre otra. El modelo es formulado como un diagrama de flujos, en el cual las flechas que conectan a las variables representan covarianzas o coeficientes de regresión (Asher, 1991). Los coeficientes de regresión representan la relación entre una variable y otra. A través del análisis de trayectorias, se especifica el modelo. Mediante este proceso, el investigador establece las relaciones directas e indirectas entre las variables. Para determinar si los datos respaldan el modelo teórico bajo prueba, se establecen indicadores de Bondad de ajuste. En el caso de EQS se establece un indicador estadístico que es la chi cuadrada $\left(\mathrm{X}^{2}\right)$ y los indicadores prácticos que controlan el efecto del número de sujetos sobre la significatividad de la comparación. Estos indicadores son el Índice Bentler-Bonett de Ajuste Normado (IAN), el Índice BentlerBonett de Ajuste No Normado (IANN) y el Índice de Ajuste Comparativo (IAC). Dado que en nuestro caso estamos probando si el modelo teórico está respaldado por los datos $\longrightarrow$ sea que el modelo propuesto no es significativamente diferente de los datos recogidos- entonces esperaríamos que el valor de la $\mathrm{X}^{2}$, que estima la relación modelo-datos, sea bajo y no significativo; es decir que su probabilidad asociada sea mayor a .05. Para los 
indicadores prácticos se requiere que su valor se acerque a 1.0 , obteniendo al menos un valor de .90 , como evidencia de bondad de ajuste (Bentler, 1993).

\section{Resultados}

Análisis univariados y confiabilidad de las escalas. El Cuadro 2 muestra los análisis de confiabilidad, medias y desviaciones estándar, así como los puntajes mínimos y máximos que produjeron las variables que constituyeron las escalas en el estudio. Puede observarse que las alfa fueron al menos de .60 para todas las escalas, lo cual es un indicador de su confiabilidad.

\section{Cuadro 2}

Medias y confiabilidad de escalas utilizadas en el estudio

\begin{tabular}{|lccccccc|}
\hline Escala y variables & N & Min. & Max. & Media & DE & alfa \\
\hline Asertividad & 194 & 0 & 4 & 2.33 & 1.42 & .70 \\
Pienso y expreso con facilidad ideas y emociones & 194 & 0 & 4 & 2.65 & 1.41 & \\
Yo soy capaz de controlar & 194 & 0 & 4 & 1.86 & 1.51 & \\
Trato de tomar cargos en situaciones & 194 & 0 & 4 & 1.92 & 1.36 & \\
Puedo hablarle a la gente con facilidad & 194 & 0 & 4 & 2.80 & 1.33 & \\
Es fácil decir lo que yo quiero & 194 & 0 & 4 & 2.45 & 1.41 & \\
Puedo decir lo que pienso & 194 & 0 & 4 & 2.48 & 1.40 & \\
Yo hablaría enfrente personas & 194 & 0 & 4 & 1.95 & 1.49 & \\
Inicio conversaciones & 194 & 0 & 4 & 2.25 & 1.40 & \\
Hago amistades fácilmente & 194 & 0 & 4 & 2.62 & 1.37 & \\
Abuso del padre & 196 & 0 & 6 & 0.69 & 1.49 & .76 \\
Papá me grita & 196 & 0 & 6 & 1.12 & 1.75 & \\
Papá amenaza con pegarme & 196 & 0 & 6 & 0.76 & 1.54 & \\
Papá me llama tonto & 196 & 0 & 6 & 1.07 & 1.79 & \\
Papá me dice me correrá a golpes & 196 & 0 & 6 & 0.20 & 0.94 & \\
Papá me pega en la cara & 196 & 0 & 6 & 0.30 & 1.14 & \\
Abuso de la madre & 196 & 0 & 6 & 0.76 & 1.40 & .73 \\
Mamá me grita & 196 & 0 & 6 & 1.40 & 1.85 & \\
Mamá amenaza con pegarme & 196 & 0 & 6 & 0.75 & 1.49 & \\
Mamá me llama tonto & 196 & 0 & 6 & 1.15 & 1.76 & \\
Mamá me dice me correrá a golpes & 196 & 0 & 6 & 0.20 & 0.93 & \\
Mamá me pega en la cara & 196 & 0 & 6 & 0.24 & 0.98 & \\
\hline
\end{tabular}


Predictores familiares y conductuales de la problemática escolar en alumnos...

Cuadro 2 (continuación)

\begin{tabular}{|c|c|c|c|c|c|c|}
\hline Escala y variables & $\mathrm{N}$ & Min. & $\mathrm{M}$ & Media & $\mathrm{DE}$ & alfa \\
\hline Violencia entre padres & & & & & & .91 \\
\hline Papá insulta con malas palabras a mamá & 203 & 0 & 6 & 1.01 & 1.77 & \\
\hline Mamá insulta con malas palabras a papá & 203 & 0 & 6 & 0.82 & 1.59 & \\
\hline Papá le tira con cosas a mamá & 203 & 0 & 6 & 0.40 & 1.20 & \\
\hline Papá empuja o agarra violentamente a mamá & 203 & 0 & 6 & 0.38 & 1.20 & \\
\hline Mamá empuja o agarra violentamente a papá & 203 & 0 & 6 & 0.27 & 1.00 & \\
\hline Papá le pega una cachetada a mamá & 203 & 0 & 6 & 0.18 & 0.77 & \\
\hline Mamá le pega una cachetada a papá & 203 & 0 & 6 & 0.23 & 0.85 & \\
\hline Papá le pegó una patada, puñetazo a mamá & 203 & 0 & 6 & 0.26 & 1.02 & \\
\hline Mamá le pegó una patada, puñetazo a papá & 203 & 0 & 6 & 0.19 & 0.81 & \\
\hline Papá golpea a mamá durante algunos minutos & 203 & 0 & 6 & 0.26 & 1.03 & \\
\hline Papá le ha roto la ropa o destruido cosas a mamá & 203 & 0 & 6 & 0.18 & 0.79 & \\
\hline Mamá le ha roto la ropa o destruido cosas a papá & 203 & 0 & 6 & 0.24 & 0.97 & \\
\hline Familia no cooperativa & & & & & & .77 \\
\hline Un miembro toma mayoría decisiones & 204 & 0 & 24 & 8.26 & 6.46 & \\
\hline Cada uno decide sus propias cosas & 204 & 0 & 6 & 2.4 & 2.18 & \\
\hline Entramos y salimos de casa cuando queremos & 204 & 0 & 6 & 1.75 & 2.18 & \\
\hline Cuando sentimos ganas de hacer algo lo hacemos & 204 & 0 & 7 & 2.35 & 2.19 & \\
\hline Conducta antisocial & & & & & & .72 \\
\hline Salir sin permiso de la casa & 195 & 0 & 6 & 1.67 & 2.03 & \\
\hline Haber roto botellas o tirado basura & 195 & 0 & 41 & 2.06 & 6.15 & \\
\hline Haber llegado tarde a propósito a casa & 195 & 0 & 6 & 1.50 & 1.99 & \\
\hline Haberse peleado con otro & 195 & 0 & 6 & 1.63 & 2.04 & \\
\hline Entrar a una tienda cerrada, robando o sin robar & 195 & 0 & 6 & 0.14 & 0.70 & \\
\hline Robarse cosas de los autos & 195 & 0 & 6 & 0.24 & 0.93 & \\
\hline Llevarse algún arma por si peleaba & 195 & 0 & 6 & 0.35 & 1.26 & \\
\hline Planeaste entrar a una casa a robar & 195 & 0 & 6 & 0.28 & 1.15 & \\
\hline Tomaste bicicleta de un desconocido & 195 & 0 & 6 & 0.29 & 1.14 & \\
\hline Delincuencia & & & & & & .88 \\
\hline Robaste cosas de almacenes estando abiertos & 195 & 0 & 6 & 0.33 & 1.11 & \\
\hline Conseguiste dinero amenazando a personas & 195 & 0 & 6 & 0.20 & 0.88 & \\
\hline Perteneciste a alguna pandilla & 195 & 0 & 1 & 0.04 & 0.21 & \\
\hline Veces que te metiste en peleas o disturbios & 195 & 0 & 6 & 0.31 & 1.06 & \\
\hline Veces que tomaste licor o drogas & 195 & 0 & 8 & 0.40 & 1.40 & \\
\hline Veces que robaste & 195 & 0 & 9 & 0.18 & 0.90 & \\
\hline Veces que cargaste un arma blanca o de fuego & 195 & 0 & 9 & 0.34 & 1.28 & \\
\hline Veces que heriste a personas con armas & 195 & 0 & 7 & 0.23 & 1.00 & \\
\hline
\end{tabular}


Cuadro 2 (continuación)

\begin{tabular}{|c|c|c|c|c|c|c|}
\hline Escala y variables & $\mathrm{N}$ & Min & & Media & DE & alfa \\
\hline Alcohol de la madre & & & & & & .60 \\
\hline Veces que bebe alcohol los fines de semana & 203 & 0 & 9 & 0.43 & 0.94 & \\
\hline Veces que bebe alcohol entre semana & 203 & 0 & 5 & 0.24 & 0.70 & \\
\hline Cantidad que bebe en cada ocasión & 203 & 0 & 6 & 0.49 & 0.94 & \\
\hline Habilidades sociales & & & & & & \\
\hline Prestas atención y comprendes lo que te dicen & 195 & 0 & 4 & 3.05 & 1.10 & \\
\hline Hablas a otros sobre cosas que interesan a ambos & 195 & 0 & 4 & .03 & 1.06 & \\
\hline Permites que sepan que les agradeces favores & 195 & 0 & 4 & 2.90 & 1.14 & \\
\hline Dices cuando te gusta algún aspecto de otro & 195 & 0 & 4 & 2.50 & 1.21 & \\
\hline Pides ayuda cuando tienes dificultades & 195 & 0 & 4 & 2.65 & 1.12 & \\
\hline Eliges la mejor forma de integrarte a un grupo & 195 & 0 & 4 & 2.74 & 1.14 & \\
\hline Pides disculpas por haber hecho aigo mal & 195 & 0 & 4 & 2.94 & 1.12 & \\
\hline Intentas convencer a otro que tus ideas son mejores & 195 & 0 & 4 & 1.61 & 1.30 & \\
\hline Intentas reconocer tus emociones & 195 & 0 & 4 & 2.70 & 1.11 & \\
\hline Permites que conozcan lo que sientes & 195 & 0 & 4 & 2.10 & 1.21 & \\
\hline pan que te preocupas por ellos & 195 & 0 & 4 & 2.49 & 1.19 & \\
\hline rte haces algo para disminuir el miedo & 195 & 0 & 4 & 2.44 & 1.28 & \\
\hline algo que es apreciado por los demás & 195 & 0 & 4 & 2.57 & 1.06 & \\
\hline Contr & 195 & 0 & 4 & 2.36 & 1.24 & \\
\hline enes al m & 195 & 0 & 4 & 2.61 & 1.26 & \\
\hline Tienes & 195 & 0 & 4 & 2.87 & 1.23 & \\
\hline otros or & 195 & 0 & 4 & 2.61 & 1.17 & \\
\hline y tener éxito en el futuro & 195 & 0 & 4 & 2.88 & 1.07 & \\
\hline Comprendes una acusación & 195 & 0 & 4 & 2.27 & 1.30 & \\
\hline Prob & & & & & & \\
\hline Te $\mathrm{m}$ & 202 & 0 & 5 & 0.44 & 1.09 & \\
\hline Faltaste a la escuela & 202 & 0 & 5 & 1.26 & 1.25 & \\
\hline No te juntaste con alguien en la escuela & 202 & 0 & 5 & 0.96 & 1.58 & \\
\hline Participaste en actividades de tu escuela & 202 & 0 & 5 & 1.69 & 1.78 & \\
\hline Te suspendieron de la escuela & 202 & 0 & 5 & 0.42 & 1.11 & \\
\hline Dejaste de ir a la escuela & 202 & 0 & 5 & 0.40 & 1.10 & \\
\hline Te expulsaron de la escuela & 202 & 0 & 5 & 0.44 & 1.16 & \\
\hline Te cambiaron de escuela & 202 & 0 & 5 & 0.43 & 1.07 & \\
\hline Respondiste en clase en voz & 202 & 0 & 5 & 0.72 & 1.30 & \\
\hline Te costó trabajo desobedecer las reglas & 202 & 0 & 5 & 1.04 & 1.53 & \\
\hline Discutes con tus compañeros & 202 & 0 & 5 & 1.18 & 1.54 & \\
\hline
\end{tabular}

Análisis de trayectorias. La Figura 1 muestra las relaciones entre las variables familiares estudiadas y los comportamientos antisocial y 
Predictores familiares y conductuales de la problemática escolar en alumnos...

delictivo, los problemas escolares y las calificaciones de los adolescentes investigados. El maltrato al adolescente de parte de su madre y de su padre, el vivir en una familia no cooperativa y la violencia entre los padres se constituyen en generadores de conducta antisocial. Todos estos efectos explican casi la mitad (48\%) de comportamiento antisocial del joven. Este comportamiento, a su vez, impacta en el desarrollo de conducta delictiva, explicando también un $48 \%$ de la variación en la conducta delictiva. El comportamiento antisocial presenta también un efecto negativo en el promedio escolar, el cual es influido de manera positiva por las habilidades sociales del adolescente y sus niveles de asertividad. Las tres variables logran impactar en un $15 \%$ de la varianza del promedio escolar. Por otro lado, la violencia entre los padres repercute en la ingesta de alcohol de la madre y esta, a su vez, impacta en los problemas escolares del joven, problemas que se ven agravados por la conducta delictiva del adolescente. Solo las habilidades sociales tienen un efecto negativo en estos problemas, es decir, inhiben su presentación. La ingesta de alcohol de la madre, la conducta delictiva y las habilidades sociales del adolescente explican un $29 \%$ de la varianza en los problemas escolares.

Los indicadores de bondad de ajuste de este modelo muestran que los datos respaldan esta propuesta teórica de relaciones entre variables. La chi cuadrada fue de 54.01 ( $\mathrm{gl}=43, p=.12)$. Todos los indicadores de bondad de ajuste práctico también respaldan al modelo: $I A N=.90, I A N N=.97$ y $I A C=.98$. 


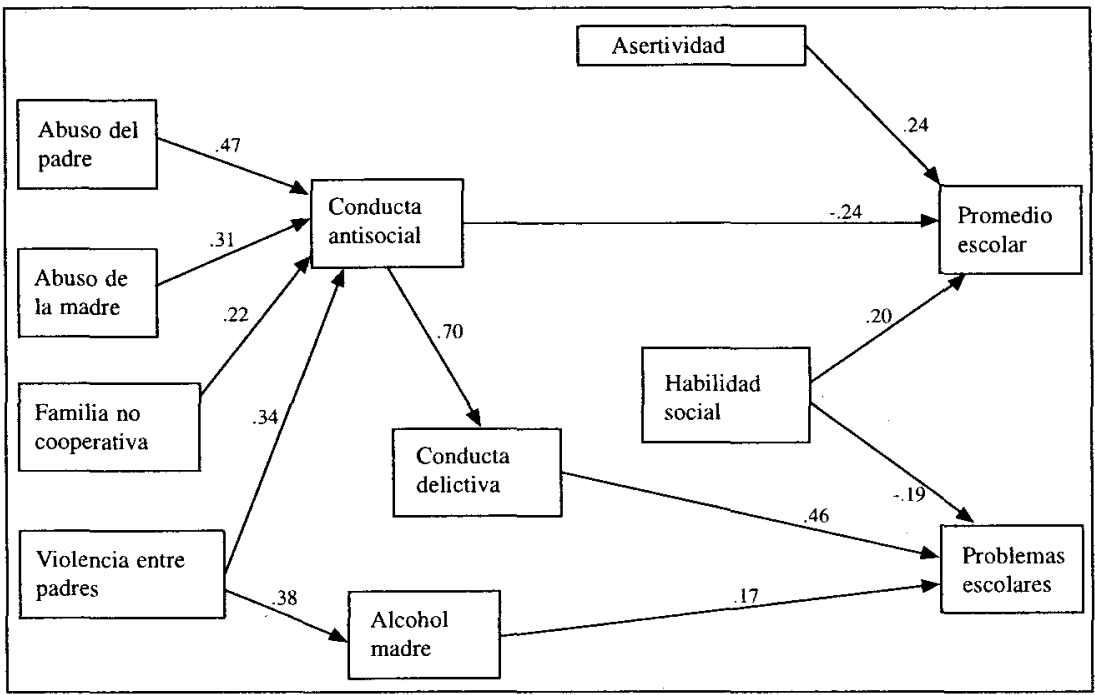

Figura 1. Efectos de variables familiares sobre la conducta antisocial y delictiva, el promedio de calificaciones y los problemas escolares en adolescentes. Todos los coeficientes estructurales son significativos $(p<.05)$. Bondad de ajuste: $X^{2}=54.01$ $\mathrm{gl}=43, \mathrm{p}=.12 ; \mathrm{IAN}=.90 ; \mathrm{IANN}=.97 ; \mathrm{IAC}=.98$.

\section{Discusión}

Los datos señalan la importancia de la familia en la conducta y el aprovechamiento escolar de los adolescentes. Los jóvenes que viven en un ambiente familiar disfuncional presentan más conducta antisocial, y esta va a influir en los problemas de conducta de los estudiantes de educación media y media superior. De acuerdo con la literatura, la dirección de la relación entre conducta escolar y antisocial no ha sido aclarada. Sin embargo, se ha encontrado consistentemente que los jóvenes que delinquen presentan problemas escolares (Anderson y Walsh, 1998).

La violencia familiar es uno de los factores que más sobresale en la predicción de la conducta delictiva. Aparentemente, el ser testigos 
Predictores familiares y conductuales de la problemática escolar en alumnos...

de la violencia del padre hacia la madre es tan perjudicial para los menores como el recibir la violencia directamente. Estos resultados están de acuerdo con los encontrados por Holden, Jennifer y Jouriles (1999). También sobresale dentro de estos efectos, la influencia que tiene el vivir en una familia en la que sus integrantes no manifiestan conductas de apoyo, ayuda o cooperación. En esas condiciones, los jóvenes se ven inclinados a desarrollar conductas antisociales, las cuales pueden desembocar en actos abiertamente delictivos. Ya planteado ese cuadro de disfuncionalidad familiar y sus efectos directos sobre la conducta antisocial y la delictiva, la cascada de efectos llega hasta el comportamiento del joven en la escuela. Los menores antisociales tienen un menor rendimiento escolar y aquellos que se involucran en actos delictivos también presentan problemas en la escuela. Dos factores individuales parecen atemperar estos efectos negativos de la familia disfuncional: La asertividad del estudiante incrementa el nivel de calificaciones, lo mismo que sus habilidades sociales, las cuales también previenen, aunque en pequeña medida su participación en acciones problemáticas de la escuela.

En resumen, estos resultados hablan del importante papel que juega la familia en el éxito o fracaso de un estudiante adolescente. Los jóvenes que van a la escuela tras convivir con padres que los maltratan, que se agreden entre sí, que esconden sus problemas tras las adicciones, y que no fomentan la cooperación entre los miembros de la familia, se ven arrastrados a reproducir esas acciones en términos de conducta antisocial y criminal, lo cual inhibe su desempeño escolar y termina por expulsarlos o marginarlos de las oportunidades educativas.

En estas circunstancias, el papel de los orientadores escolares debiera enfocarse a la detección de los problemas familiares de cada estudiante. El buscar solo en las características individuales (motivación, capacidades, hábitos de estudio, impulsividad) las fuentes del éxito o fracaso escolar de seguro no garantiza la solución cabal de 
los problemas escolares. En la familia se encuentra la base de los mismos, y hacia ella debiera encaminarse una parte sustancial del esfuerzo de intervención y prevención de esos problemas escolares.

\section{Referencias}

Angenent, H. y de Man, A. (1996). Background factors of juvenile delincuency. Nueva York: Peter Lang Publishing.

Anderson, L. E. y Walsh, J. A. (1998). Prediction of adult criminal status from juvenile psychological assessment. Criminal Justice and Behavior, 25, 226-239.

Asher, H. B. (1991). Causal modeling. Sage University Paper Series on Quantitative Application in the Social Sciences. Beverly Hills: Sage.

Bentler, P. M.'(1993). EQS, Structural Equation Program manual. Los Angeles: BMDP Statistical Software.

Bronfenbrenner, U. (1979). The ecology of human development. Cambridge, MA: Harvard University Press.

Dabas, E. (1998). Redes sociales, familias y escuela. México, DF: Paidós.

Eckenrode, J. y Doris, J. (1991). The academic effects of child abuse and neglect. Washington, DC: National Center for Child Abuse and Neglect.

Frías, M., Corral, V., Moreno, C., y Rodríguez, I. (2000). El maltrato infantil como un factor de riesgo para la salud, la conducta prosocial y la ejecución escolar en niñas y niños. En J. E. Ramos (Ed.). Investigaciones educativas en Sonora (vol.2) (pp. 32-65). Hermosillo, México: REDIES.

Frías, M., Ramírez, J. M., Soto, R., Castell, I. y Corral, V. (2000). Repercusiones del castigo corporal en niños: un estudio con grupos de alto riesgo. En Asociación Mexicana de Psicología Social (AMEPSO) (Eds.). La psicología social en México (vol. 6) (pp. 479-485). México: Autor. 
Predictores familiares y conductuales de la problemática escolar en alumnos...

Garbarino, J. y Eckenrode, J. (1999). Por qué las familias abusan de sus hijos. México, DF: Granica.

Gelles, R. J. y Straus, M. A. (1990). The medical and psychological costs of family violence. En M. A Straus y R. J. Gelles (Eds.). Physical violence in American families: risk factors and adaptations to violence in 8,145 families (pp 425-430). Nuevo Brunswick, NJ: Transaction.

Goldstein, P. A., Sprafkin, R. P., Gershaw, N. y Klein, P. (1989). Habilidades sociales y autocontrol en la adolescencia: un programa de enseñanza. Barcelona: Martínez Roca.

Grasmick, T., Tittle, G., Bursik, J. y Bruce, D. (1993). Testing the core empirical implications of Gottfredson, and Hirachi's general theory of crime. Journal of Research in Crime and Delinquency, 30, 5-29.

Gove, W. y Crutchfield, R. (1982). The family and juvenile delinquency. Sociological Quarterly, 23, 301-319.

Harter, S. (1998). The effects of child abuse on the self-system. Journal of Aggression, Maltreatment and Trauma, 2, 147-169.

Holden, G., Geffner, R., y Jouriles, E. (1999). Children exposed to marital violence. Washington, DC: American Psychological Association.

INEGI (1992). Sistema para la consulta de información censal (SCINCE). México, DF: Instituto Nacional de Estadística, Geografía e Informática.

Kazdin, A. E. (1988) Tratamiento de la conducta antisocial en la infancia y la adolescencia. Barcelona: Martínez Roca.

Kinard, E. M. (1999). Pychosocial resources and academic performance in abused children. Children and Youth Services Review, 21, 351376.

Kinard, E. M. (2001). Percibe and actual academic competence in maltreated children. Child Abuse \& Neglect, 25, 33-45.

Loeber, R. y Dishion, T. (1982). Early predictors of male delinquency: a review. Psychological Bulletin, 94, 68-99. 
Loeber, R. (1986). What policy makers and practitioners can learn from studies of juvenile conduct problems and delinquency. From children to citizens: families, schools and delinquency prevention. Nueva York: Springer.

Reich, W. y Herjanic, B. (1989). The diagnostic interview for children and adolescents (dica). Manuscrito no publicado, Departamento de Psiquiatría de la Universidad de Washington, St. Louis, MO.

Sarafino, E. P. y Amstrong, J. W. (1991). Desarrollo del niño y del adolescente. México: Trillas.

Straus, M. A. (1979). Measuring intrafamily conflict and violence: The conflict tactics scale (CTS.). Journal of Marriage and the Family, 41, 75-88.

Straus, M. A. (1980). Wife beating: How common and why? En M. A. Straus y G. T. Hotaling (Eds.). The social causes of husband wife violence (pp. 23-38). Minneapolis: University of Minnesota Press.

Straus, M. A. (1990). The conflict tactics scale and its critics: An evaluation on new data on validity and reliability. En M. A. Straus y R. J. Gelles (Eds.). Physical violence in American families. Nuevo Brunswick, N J: Transaction.

Straus, M.A.(1991). Discipline and deviance: Physical punishment of children and violence in adulthood. Durham: University of New Hampshire-family Research Laboratory.

Straus, M., Hamby S., Finkelhor, D., Moore, D. y Runyan, D. (1998). Identification of child maltreatment with the parentchild conflict tactics scales: development and psychometric data for a national sample of American parents. Child Abuse \& Neglect, 22, 249-270.

Wells, L. y Rankin, J. (1986). Self-concepts as a mediating factor in delinquency. Social Psychology Quarterly, 46, 11-22. 